\title{
Oral Biotherapeutics- A Review Article
}

\author{
1Shikha Mali, ${ }^{2}$ Shilpy Singla, ${ }^{3}$ Shilpi Tiwari, ${ }^{4}$ Nikita Agarwal, ${ }^{5}$ Sumedha Srivastava, 6 Pramod \\ Yadav \\ ${ }^{1}$ Senior Lecturer, Department of Paedodontics and Preventive Dentistry, People's College of Dental \\ Sciences And Research Centre, Bhopal, Madhya Pradesh, India. \\ 2Professor \& Head, Department of Pedodontics and Preventive Dentistry, Index dental college, tilak path, \\ Indore, Madhya Pradesh, India. \\ ${ }^{3}$ Reader, Department of Paedodontics and Preventive Dentistry, People's College of Dental Sciences And \\ Research Centre, Bhopal, Madhya Pradesh, India. \\ ${ }^{4}$ Reader, Department of Paedodontics and Preventive Dentistry People's College of Dental Sciences And \\ Research Centre, Bhopal, Madhya Pradesh, India. \\ ${ }^{5}$ Reader, Departmentof Periodontics, People's College of Dental Sciences And Research Centre, Bhopal, \\ Madhya Pradesh, India. \\ ${ }^{6}$ Assistant professor, Department of Periodontics and community dentistry, Dr. Z. A dental college, AMU \\ Aligarh, India.
}

Review article

Address for Correspondence Author

Dr. Shikha Mali; Department of Paedodontics And Preventive Dentistry, People's College of Dental Sciences

And Research Centre, Bhopal, Madhya Pradesh, India.

E-mail: shikha9290@gmail.com

Crossref doi: https://doi.org/10.36437/ijdrd.2020.2.2.S

\section{ABSTRACT}

Probiotics, live cells with different beneficiary characteristics, have been extensively Studied and explored commercially in many different products in the world. Their benefits to human and animal health have been proven in hundreds of scientific research. Lactobacillus and Bifidobacterium are the main probiotic groups; however, there are reports on the probiotic potential of Pediococcus, Lactococcus, Bacillus, and yeasts. Some of the identified probiotic strains exhibit powerful anti-inflammatory, antiallergic, and other important properties.

Keywords: Probiotics, Intestinal Microflora, Bifidobacterium, Lactobacillus, Immune Stimulation, Probiotic Production.

\section{Introduction}

In the people of all ages, the changing food habits and lifestyle have resulted in deterioration of oral health. The increasing global problems with traditional disease management strategies have prompted the investigators to hunt for better and new alternatives to deal with oral health issues. In the past few years, the global demand for chemical-free, less harmful, and easier solutions to oral health problems have increased. The most promising 
alternative to traditional disease management is those viable microorganisms which are constituents of the natural microflora of the human body. According to the World Health Organization, "Probiotics are live microorganisms which, when administered in adequate amounts, confer a health benefit on the host."1

The risk of colonization by oral pathogens can be decreased by Probiotic therapy without depleting the friendly microflora. Probiotics are readily incorporated in the natural microflora of the human body and resemble the human body microbiota. They are harmless easy to consume and in many edible forms (such as cheese, yoghurt, etc.).

The inability of the antibiotics to discriminate good bacteria from the disease-causing bacteria, the increasing rate of antibiotic-associated side effects, the development of antimicrobial-resistant mutants, and their complications suggests an urgent need to switch our therapeutic approach from traditional antibiotics to the probiotic therapy for oral care.

The first part of the gastrointestinal tract is a mouth. In the human mouth, more than 700 species of oral microbiota have been detected. The important functions of Resident microbiota such as reducing the susceptibility to pathogen attack, developing immune response against pathogens and prevention of pathogen colonization. ${ }^{2}$ These bacteria reside either integrated into the oral biofilm or in the planktonic state. Saliva is a complex medium in the mouth that contains different bacteriostatic, bactericidal, and inhibitory proteins that collectively may damage a variety of species in the planktonic state. Ingested Probiotics are first exposed to the salivary proteins such as lactoferrin, histatin, lysozyme, salivary peroxidase, secretory IgA, and cystatins, which affects the adhesion, metabolic activity, morphology and viability of the probiotic microorganism. On the other hand, Saliva tends to propagate oral biofilm and contributes to the microbial diversity in the mouth. The continuous salivary flow in the oral cavity can lead to detachment of some microbes from the biofilm surfaces, modulating the microbial colonization. Selecting Probiotics for the oral cavity different strains show specific response to proteolytic enzymes and this strain-specific response need to be considered. Many bacterial species have been found to survive within buccal epithelial. Culture-based studies suggest that bifidobacteria are among the first anaerobes in the oral cavity. The first microorganisms to be exposed to an oral cavity of an infant are lactobacilli and Bifidobacterium as they are present in breast milk. ${ }^{3}$

The most common probiotic strains belong to the genera lactobacillus and Bifidobacteria. Probiotics first compete with the oral pathogens for the adhesion site and then colonizes the oral surface. After the probiotics aggregate on the oral surface, they compete with oral pathogens for nutrients, growth factors and also produce antimicrobial compounds, including hydrogen peroxide, carbon peroxide organic acids, diacetyl, low molecular weight antimicrobial substances, adhesion inhibitors, and bacteriocins. Probiotics can also activate and modulate the immune system, and they have been shown to reinforce gut defence by immune exclusion, immune elimination, and immune regulation .4

\section{Probiotic Microorganisms}

Currently used probiotic microorganisms in the treatment of oral diseases are Lactobacilli species, Bifidobacterium species, Streptomyces species. Lactobacilli and Bifidobacteria have been reported as promising bacteria for the prevention of dental caries.

Lactic acid bacteria (LAB) are facultative anaerobes, non-sporulating, and acid tolerant strictly fermentative and they may possess potential therapeutic properties including anticancer and anti-inflammatory activities. The genus Lactobacillus currently consists of over 180 species and encompasses a wide variety of organisms. 
Some of the species are L. acidophilus, L. rhamnosus, L. johnsonii, L. casei, L. gasseri, L. fermentum, L. plantarum, L. brevis, L. lactis, L. reuteri, L. paracase. Nase et al became the first researcher to investigate the inhibition of caries pathogens using lactobacillus strain, L. rhamnosus CG. He concluded Supplementing 1-6 year old children with L. rhamnosus for 7 months significantly reduced the risk of dental caries. Ahola et al in 2002 found that Lactobacillus gasseri when ingested in the form of probiotic dairy product reduced the Streptococcal mutans count in saliva of adults and showed prevention of caries in children. ${ }^{5}$ L. reuteri has also been investigated for its caries preventing effects. L.reuteri has been reported to produce water-soluble, broadspectrum antimicrobial compounds that exhibit antagonistic activity such as reuterin and reutericyclin. These compounds are resistant to proteolytic and lipolytic enzymes and are active over a wide range of PH. According to Chung et al, $2004 \mathrm{~L}$. fermentum was found in the saliva of healthy children. ${ }^{6}$ This strain significantly inhibited the formation of the insoluble glucan produced by S. mutans. It did not affect the multiplication of this pathogenic strain, but it completely inhibited the adherence onto cuvette walls. Sookkhee and coworkers screened 3790 lactic acid bacteria for their ability to inhibit the in vitro growth of various oral pathogens. L. paracasei and L. rhamnosus were found to have potent antimicrobial activity against a number of oral pathogens. ${ }^{7}$ Lactobacillus lactis was reported to modulate the growth of oral microflora, eliminating the colonization of Streptococcus oralis, Veillonela dispar, Actinomyces and carcinogenic Strep. sobrinus. Stamatova et al, 2007 used L. rhamnosus \&L. bulgaricus strain and concluded that Inhibitory effects against P. gingivalis, Fusobacterium nucleatum \& streptococcal spp. ${ }^{8}$

Chewing Gum "perio balance" is the first probiotic specifically formulated to fight periodontal disease. It contains a combination of two strains of L. reuteri, specially selected for their synergetic properties in fighting cariogenic bacteria and periodonto-pathogens. Pozharitskaia et al gave the Tablet 'Acilact' containing $L$. acidophilus which showed improvement in clinical parameters along with shift in local microbiota towards Gram +ve cocci and lactobacilli. ${ }^{9}$ Krasse et al studied on chewing gum containing L. Reuter iand reported reduction in gingivitis. ${ }^{10}$ Consumption of probiotic milk drink containing Lactobacillus Casei reduced the MMP3 , elastase activity in students with plaque induced gingivitis. It has been reported that lactobacillus brevis prevents the production of nitric oxide, the release of prostaglandin E2 (PGE2) and the activation of MMPs, thus reducing bone resorption associated with periodontitis. During the fermentation process in milk, Lactobacillus helveticus produces short peptides that act on osteoblasts and increase their activity in bone formation. Hatakka K et al (2007) reported decreased high oral yeast count in individuals having cheese containing containing L. rhamnosus strains GG. ${ }^{11}$

Bifidobacterium is a genus of Gram-positive, non-motile, often branched anaerobic bacteria. Some Bifidobacterium strains are considered as important probiotics and used in the food industry. One approach has been the addition of live cultures to food stuffs such as fermented milk products (e.g., yogurts).The various species of Bifidobacterium used in treatment of oral diseases are B. bifidum, B. longum, B. infantis, Bifidobacterium DN-173 010 B. adolescentis, B. animalis B. thermophilum. Caglar and his co-workers have studied the effects of bifidobacteria on oral health. A semi-quantitative diagnostic kit determining the salivary count of S. mutans and lactobacilli showed a decrease in salivary S. mutans count in the bifidobacterium containing yoghurt with no effect on lactobacillus count.12 Grudianov et al. in 2002 carried out a clinical study where they obtained a probiotic mix in the tablet forms, Acilact and Bifidobacterium, which produced normalization of microflora with reduction in signs of gingivitis and periodontitis. ${ }^{13}$ Çaglar, Sandalli, Twetman, et al.in 2005 initially demonstrated that once daily consumption of yogurt containing Bifidobacterium DN173010 for a period of 14 days led to a significant reduction in the number of the salivary mutans streptococci but no significant reduction in lactobacilli.12 More recently Cildir, et al. 2008 investigated the effect of once daily probiotic Bifidobacterium DN-173010 yogurt consumption in orthodontic patients and demonstrated a 
significant reduction of salivary mutans streptococci after 14 days, but could not demonstrate a significant change in salivary lactobacilli counts. ${ }^{14}$ Certain cellular components of bifidobacteria act as immune-modulation promoting immunological attack against malignant cells. Other species used as probiotic microorganisms are Streptococcus. salivarius, Streptococcus thermophilus, W.sibaria, Leuconostoc, Pediococcus, Propionibacterium. C. albicans, Sacchromyces boulardii, A. niger, A. oryzae, c. pintolopesii.

Streptococcus salivarius is a species of spherical, Gram-positive bacteria which colonize the mouth and upper respiratory tract of humans a few hours after birth, making further exposure to the bacteria harmless in most circumstances. Some strains of S. salivarius are being trialed for their use as a probiotic in the prevention of oral infections and it was detected most frequently among people without halitosis therefore considered a commensal bacterium of the oral cavity. Many of the compounds that contribute to oral malodor are volatile sulfur compounds (VSCs), such as hydrogen sulfide (H2S), methyl mercaptan (CH3SH), and dimethyl sulfide (CH3SCH3). Kazor et al produces bacteriocins which reduces the number of bacteria that produce Volatile Sulphur Compounds. ${ }^{15}$ S. Salivarius also suppress volatile sulfide effects, by competing for colonization areas with volatile sulfide-producing Species. The use of gum or lozenges containing S. salivarius K12 reduced the levels of VSCs among patients diagnosed with halitosis. Periodontopathic bacteria, including Porphyromonas gingivalis, Treponema denticola, Prevotella intermedia, and Fusobacterium nucleatum, produce H2S and CH3SH.

Streptococcus thermophilus is one of the most widely used bacteria in the dairy industry. S. thermophilus is found in fermented milk products and generally used in the production of yogurt along with Lactobacillus delbrueckii subsp. bulgaricus. Streptococcus thermophilus and Lactococcus lactis with the ability to incorporate into the biofilm similar to the dental plaque and grow along with five other strains of oral bacterial species associated with supragingival plaque.

Presence of S.oralis and S. uberis provides a good indication of health of periodontium. Kang et al in 2006 reported Weilonella cibari used for gargling; it led to marked reduction in the production of hydrogen sulphide and methanethiol and reduction in foul smell. Hyink et al found Atopobium parvulum, Eubacterium sulci and Solobacterium moorei predominate on the dorsal surface of the tongue among people with halitosis and reduces halitosis. ${ }^{16}$ Resident microbiota performs several functions, benefits health and shields the body from various pathogenic microorganisms. Caglar et al. in 2006 studied the residence time of probiotics in oral cavity after withdrawal of probiotic treatment. Two-week use of a L. reuteri enriched yogurt showed a reduced S. mutans level in oral cavity. The effects were observed during use and for a few days after discontinuation. Wolf et al. in 1995 observed the loss of L. reuteri colonization; two months after having probiotic use discontinued. ${ }^{17}$ Yli-Knuuttila et al. in 2006 studied L. rhamnosus colonization in oral cavity and concluded that permanent colonization in oral cavity was unlikely in most cases. Therefore, regular use of probiotics was suggested. ${ }^{18} \mathrm{Horz}$ et al.in 2007 assessed the Latency period of probiotic S. salivarius K12 and assessed that gradual reduction in S. salivarius level beginning 8 days after treatment withdrawal. However, probiotic may be found on oral mucous membrane, tongue and in stimulated saliva for more than 3 weeks. ${ }^{19}$

Future studies have to be aimed at not only identification of the Oral microorganism that is best suited to oral biotherapy but also the most appropriate dose and vehicle for its dispensation.

Oral biotherapy using living bacteria that have beneficial characteristics-is the perfect metaphor for this new approach. Although positive results have been obtained by some researchers, a significant amount of long term research needs to be carried out before Oral biotherapy can be advocated for every patient. 


\section{References}

1. Probiotics in food: health and nutritional properties and guidelines for evaluation. Food and Agriculture Organization of the United Nations: World Health Organization, 2006.

2. Aas JA, Griffen AL, Dardis SR, Lee Am, Olsen I, Dewhirst FE et al. Bacteria of dental caries in temporary and permanent teeth in children and young adult. J Clin Microbial 2008; 46:1407-14017. https://doi.org/10.1128/JCM.01410-07

3. Parvez S, Malik KA, Ah Kang S, Kim HY. Probiotics and their fermented food products are beneficial for health. J of Appl Microbiol 2006, 100: 1171-1185. https://doi.org/10.1111/j.1365-2672.2006.02963.x

4. Isolauri E, Majamaa H, Arvola T, Rantala I, Virtanen E, Arvilommi H. Lactobacillus casei strain GG reverses increased intestinal permeability induced by cow milk in suckling rats. Gastroenterology 1993; 105: 1643-1650. https://doi.org/10.1016/0016-5085(93)91059-Q

5. Ahola AJ, Yli-Knuuttila H, Suomalainen T, Poussa T, Ahlstrom A, Meurman JH, Korpela R. Short term consumption of probiotic containing cheese and its effect on dental caries risk factors. Arch Oral Biol 2002; 47: 799-804. https://doi.org/10.1016/S0003-9969(02)00112-7

6. Chung J, Ha ES, Park HR, Kim S. Isolation and characterization of Lactobacillusspecies inhibiting the formation of Streptococcus mutans biofilm. Oral Microbiol Immunol 2004; 19(3): 214-6. https://doi.org/10.1111/j.0902-0055.2004.00137.x

7. Sookkhee S, Chulasiri M, Prachyabrued W. Lactic acid bacteria from healthy oral cavity of Thai volunteers: inhibition of oral pathogens. J Appl Microbiol 2001; 90: 172-179. https://doi.org/10.1046/j.1365-2672.2001.01229.x

8. Stamatova I, Kari K, Meurman H. In Vitro evaluation of antimicrobial activity ofputative probiotic lactobacilli against oral pathogens. Intl. J. Probiotics and Prebiotics 2007; 2(4): 225-232.

9. Pozharitskaia MM, Morozova LV, Melnichuk GM, Mel'nichuk SS. The use of the new bacterial biopreperation Acilact in the combined treatment of periodontitis. Stomatologiia (Mosk) 1994; 73:1720. https://pubmed.ncbi.nlm.nih.gov/9612040/

10. Krasse P, Carlsson B, Dahl C, Paulsson A, Nilsson A, Sinkiewicz G. Decreased gum bleeding and reduced gingivitis by the probiotic Lactobacillus reuteri. Swed Dent J. 2006; 30(2):55-60. https://pubmed.ncbi.nlm.nih.gov/16878680/

11. Hatakka K, Ahola AJ, Yli-Knuuttila H, Richardson M, Poussa T, Meurman JH, et al. Probiotics reduce the prevalence of oral candida in the elderly. A randomized controlled trial. J Dent Res 2007; 86:125-130. https://doi.org/10.1177\%2F154405910708600204

12. Caglar E, Sandalli N, Twetman S, Kavaloglu S, Ergeneli S, Selvi S. Effect of yogurt with Bifidobacterium DN-173 010 on salivary mutans streptococci and lactobacilli in young adults. Acta Odontol Scand 2005; 63: 317-320. https://doi.org/10.1080/00016350510020070

13. Grudianov AI, Dimitrieva NA, Fomenko EV. Use of probiotics Bifidumbacterin and Acilact in tablets in therapy of periodontal inflammation. Stomatologiia (Mosk) 2002; 81:39-43. https://pubmed.ncbi.nlm.nih.gov/11889866/

14. Cildir SK, Kuvvetli SS, Sandalli N. A probiotic lozenge administered medical device and its effect on salivary mutans streptococci and lactobacilli. International Journal of Paediatric Dentistry 2008; 18:3539. https://doi.org/10.1111/j.1365-263X.2007.00866.X

15. Kazor CE, Mitchel PM, Lee AM, Stokes LN, Loesche WJ, Dewhirst FE, et al. Diversity of bacterial populations on the tongue dorsa of patients with halitosis and healthy patients. J Clin Microbiol 2003; 41:558-563. https://dx.doi.org/10.1128\%2FJCM.41.2.558-563.2003

16. Hyink O, Wescombe PA, Upton M, Ragland N, Burton JP, Tagg JR. Salivaricin A2 and the Novel Lantibiotic Salivaricin B are encoded at Adjacent Loci on a 190-kilobase Transmissible Megaplasmid in 
the Oral Probiotic Strain Streptococcus Salivarius K12. Appl Environ Microbiol. 2007; 73(4):1107-13. https://dx.doi.org/10.1128\%2FAEM.02265-06

17. Wolff L, Dahlen GG, Aeppli DM. Bacteria as risk markers for periodontitis. J Periodontol 1994; 65: 498510. https://doi.org/10.1902/jop.1994.65.5s.498

18. Yli-Knuuttila H, Snäll J, Kari K, Meurman JH et al. Colonization of Lactobacillus rhamnosus GG in the oral cavity. Oral Microbiol Immunol 2006; 21:129-31. https://doi.org/10.1111/j.1399-302x.2006.00258.x

19. Horz HP, Meinelt A, Houben B, Conrads G. Distribution and persistence of probiotic Streptococcus salivarius $\mathrm{K} 12$ in the human oral cavity as determined by real-time quantitative polymerase chain reaction. Oral Microbiol Immunol 2007; 22:126-30. https://doi.org/10.1111/j.1399$\underline{302 X .2007 .00334 . x}$

How to cite this Article: ${ }^{1}$ Shikha Mali, ${ }^{2}$ Shilpy Singla, ${ }^{3}$ Shilpi Tiwari, ${ }^{4}$ Nikita Agarwal, ${ }^{5}$ Sumedha

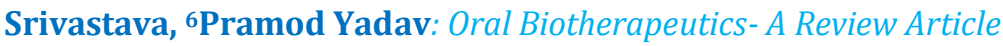
Int. J. Drug Res. Dental Sci., 2020; 2(2):30-35.

Crossref doi: https://doi.org/10.36437/ijdrd.2020.2.2.S

Source of Support: Nil, Conflict of Interest: Nil.

Received: 26-5-2020 Revised: 18-6-2020 Accepted: 27-6-2020 